\title{
Analysis of Invalidation Mechanism of Shell-Cup in Rotary Feed Mechanism
}

\author{
Guo-Hui WANG ${ }^{1}$, Peng-Fei FAN ${ }^{1, a}$ \\ ${ }^{1}$ Department of Arms Engineering, Academy of Armored Force Engineering, Beijing 100072, China
}

Keywords: Ramming machine, Modeling and simulation; Modal analysis.

\begin{abstract}
As one of new generation equipment in China, the main task of one type infantry fighting vehicle is to complete the breakthrough in the battle accompanying the main tank. It has not only the stronger firepower but equipped with auto-loader that is able to raise the fire rate and shrink the outer shape of the vehicle. However, in the practical operation of armored forces and relevant units, it found that the fault rate of this type of auto-loader is higher in some extent. And the shell-cup in rotary feed mechanism is one reason of the fault of feeding action. This text is to analyze the process and mechanism of feeding in the auto-loader in details, and simplify and assume the model of shell-cup according to its working state, then in different circumstances we analyze the strain and stress condition of it based on simulation testing dates to propose several reasonable and practicable advice, which is for the optimization design of shell-cup.
\end{abstract}

\section{Introduction}

One type of tracked infantry fighting vehicles for a new generation of heavy tracked infantry fighting vehicles in China have been fielded, it has more powerful firepower: it is able to launch $100 \mathrm{~mm}$ shell and gun launched missile, $30 \mathrm{~mm}$ shell and other major ammunition, and have certain anti-tank combat capability[1]. In order to reduce the contour and improve weapon firing rate, this type of infantry fighting vehicles equipped with new automatic loading system, to meet the general ammunition loading at the same time, also integrated the function of gun launched missile loading .

In actual using, we found that the auto-loader, who plays a key role in the weapon system performance, has a relatively high failure rate, and it is accounted for about 15\% [2] in the weapon system. Different from other systems, once the fault of automatic loading machine happened, the whole launching process will be stopped. Therefore, auto-loader fault will greatly reduce its performance in combat and security. We can analyze its failure mechanism, optimization through certain means and provide a reference for the troops and military departments.

The failure of the shell cup is one of the reasons for the failure of the feeding of ammunition, which the current forces reflect more relatively [3]. This text is going to regard the shell cup of $100 \mathrm{~mm}$ auto-loader as the research object, and simplify and assume the model of shell-cup according to its working state, then via the modeling and simulation technology, in different circumstances we can analyze the strain and stress condition of it based on simulation testing dates to propose several reasonable and practicable advice, which is for the optimization design of shellcup.

\section{Structure Principle Analysis}

A type of tracked infantry fighting vehicle gun auto-loader, consists of revolving ramming machine, feeding machine and gun launched missile ammunition mechanism. The revolving ramming machine is used for placing $100 \mathrm{~mm}$ shells and gun launched missiles. The loading system can realize the semi-automatic, fully automatic loading with fixed angle and manual loading, as well as the semi-automatic loading of the gun launched missile.

\section{For the Process Analysis}

The ramming machine by turning $100 \mathrm{~mm}$ ammunition or artillery missiles accurately conveyed to the filling position, is mainly composed of a manual transmission system, chassis, ring frame, 
missile cup cylinder, shell cup and brake. The annular rotating frame is for mounting shell cup and missile cup, ramming machine, and the main structure is shown in figure 1.

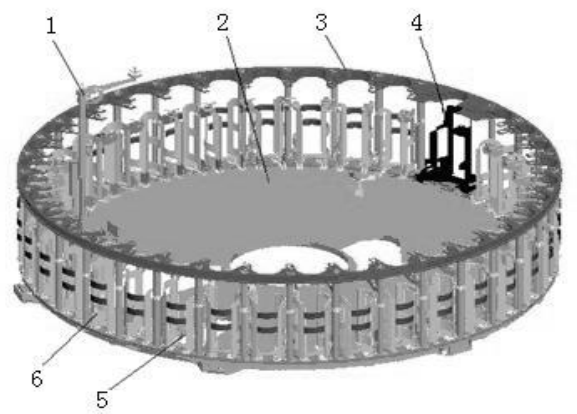

Figure 1. Schematic Diagram of the Revolving Ramming Mechanism.

The shell cup is used to place the 100mm killing explosive bomb and is fixed reliably, and the shell is transported to the designated position by the transmission device of the transmission device. Shell cup mainly comprises a cup body, a latch, rocker arm, core shaft, and crank lever, etc.. As shown in figure 2.

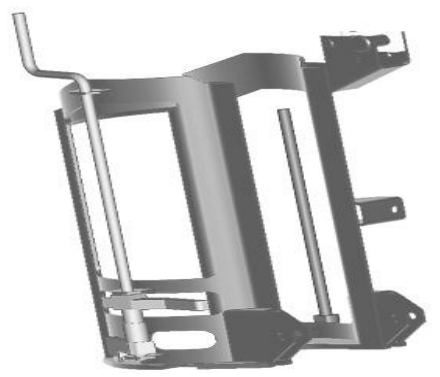

Figure 2. Schematic Diagram of Shell Cup.

The ramming machine rotation is realized by ramming elector-mechanical transmission device or a manual transmission device in the ramming process, due to the rotation between the cup and the bottom of the cylinder shell and a rotating frame, it will cause frequent collisions between the two, when the load is more intense deformation and damage degree of collision, higher probability.

When the shell reaches the specified feeding position, the switch and the latch body contact receives the corresponding signal and transmitted to the automatic control device, feeder hook arm will pull shell cup tilted to the loading angle of ammunition and pushing the bullet into the chamber action. When the shell is moving to the gun bore, shell cup remains in the original position and the inclined angle is constant.

\section{Stress Analysis}

Via Analyzing the working principle and main structure of auto-loader, we can acquire the movement of the shell cup and the stress situation, mainly including: shell cup core barrel shaft spring on a circular cylinder body spring force $\mathrm{F}_{\text {spring }}$ support base on rotating frame of the cup body $\mathrm{F}$, cup body around the rotating frame base hole rotation of the centripetal force to $\mathrm{F}$.

Here are three cases to elaborate:

(1) When the shell cup tube is in the static state, the G of the shell is subjected to the load of the

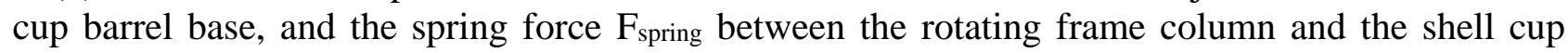
core shaft is zero;

(2) When the shell cup is in a bomb motion, gravity bomb also comprises a base shell G, the bottom of the shell cup is subjected to a periodic force, and the cup body slightly rotates around the rotating frame base hole. Rotating frame column and shell cup spring force between the cylinder 
core shaft changes at the same time, the change of $F_{\text {spring }}$ with the rotation angle of a circular cylinder by the centripetal force to the overall $\mathrm{F}$, provided by the contact force;

(3) When the shell cup is in the state of no load, it is not affected by the gravity of the shell, but with the increase of the rotation angle, the change range of the size and the direction of the spring force $F_{\text {spring }}$ increases.

\section{Model Building and Verification}

\section{Basic Assumptions}

By analyzing the motion of the rotating frame and the shell cup, the following assumptions can be made:

(1)The circular hole of the bottom of the shell cup is connected with the circular hole of the rotating frame base through a rotating pair;

(2)The shell cup body is a flexible body structure;

(3)There is a small gap between the shell and the shell cup, assuming that the pressure between the side surface of the shell and the shell body is zero;

(4)In the static state, the assumption is that the spring force between the rotating frame column and the core shaft of the shell cup is zero;

(5)In the state of motion, assuming that the vehicle has a regular vibration.

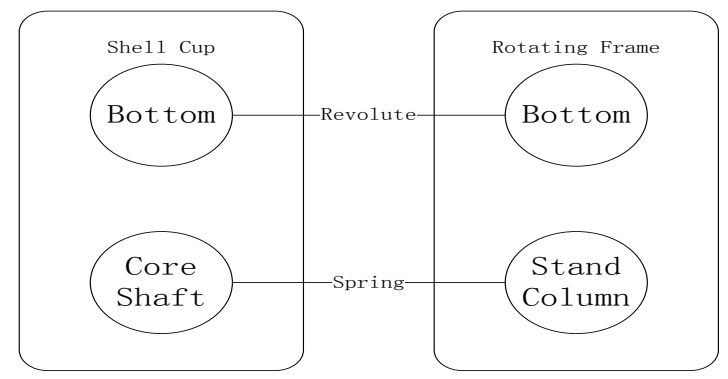

Figure 3. Connection Diagram.

On the basis of the original model, the corresponding simplification, mainly including the following aspects:

(1)The original ramming machine base is integrally formed, whose structure is more complicated, and it is not suitable for the analysis of a single shell cup. Through simplifying process with the basics to realize the rotation around its axis and provide support force. Simplified base schematic is shown in Figure 4:

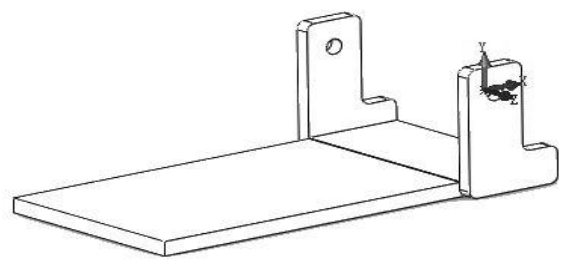

Figure 4. Simplified Base.

(2) A spring force between the column and the core shaft, but the stress point is not easy to choose, so the bottom of the mandrel force correction for round face, close to the cylinder wall and the bottom seat.

\section{Implementation Plan}

When the auto-loader is not revolving ramming, it can be divided into two circumstances: if the vehicle is stationary, shell cup is only subjected to the effect of gravity from shells, perpendicular to 
the direction of the base, and the size of $\mathrm{F}=\mathrm{G}$ constant; if the movement of the vehicle, a circular cylinder under the action of the same artillery shells from gravity, but the direction will happen a slight change of angle, the $\mathrm{F}$ bottom changes size. We defined range of its direction according to $0^{\circ} \leq \Delta \theta \leq 1^{\circ}$, and in details is shown as below:

$$
\Delta \theta=\left\{\begin{array}{l}
t^{2}-2 t+1,0<t \leq 1 \\
-\left(t^{2}-2 t+1\right), 1<t \leq 2
\end{array}\right.
$$

When the automatic loading machine of revolving ramming, if it is in the idle state, then the shell cup tube from circular motion of the centripetal force of $\mathrm{F}$ to function (from the bottom of the rotary pair contact force provided, but the direction) will change slightly larger angle, it is assumed that the scope of the definition of the change of direction angle according to $0^{\circ} \leq \Delta \theta \leq 2^{\circ}$; if at the loading state shell, a circular cylinder by the centripetal force from projectile gravity and circular motion (from the bottom of the rotary pair provides points of contact), direction will slightly change of angle, make the F bottom size changes, it is assumed that the scope of the definition of the change of direction angle according to $0^{\circ} \leq \Delta \theta \leq 1^{\circ}$. In the loading process, the turning angle is represented by a linear change of torque, while the vibration state of the vehicle is represented by a pair of force.

\section{Model Validation}

The $\mathrm{x}$-t file format is introduced into the ADAMS to verify the dynamic characteristics of the assembly model of the simplified shell cup and the base. A torque applied to a circular cylinder, and in a short period of time due to its rotation, which simulate the motion characteristics of the vehicle vibration of a circular cylinder shell, turning torque set by the STEP function in ADAMS, here is set to:

$$
\text { step }=(\text { time }, 0,60,1.5,30)
$$

Select the maker PART2_Marker6 in a circular cylinder, to analyze the X direction and speed, numerical calculation and actual model after comparing the results shown in figure 5.

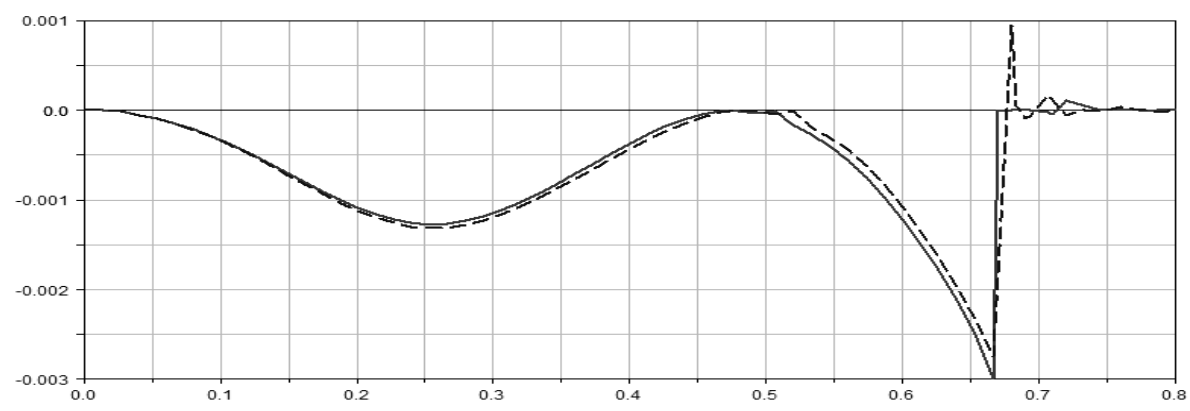

Figure 5. Comparison of Calculated Results.

We can see that in the vibration simulation process simplified model and original model, dynamic characteristics of PART2_Marker6 is basically the same, the validation of the simplified model availability, convenient for in-depth analysis of the next step.

\section{Modal Analysis}

Using ANSYS Workbench 15.0 static structure analysis module, we are able to realize the simplified simulation of shell cup with different movement and load state. The analysis process mainly includes 3D model import, meshing, loading and post-processing (calculation and results) etc..

\section{Pre-Treatment}

Pre-processing includes two steps: model import and mesh generation: 
(1)The source model is a type of tracked infantry fighting vehicle $100 \mathrm{~mm}$ gun auto-loader shell cup 3D model. The original Igs format can be imported into Solidworks, which transfers into the solid parts and be saved in sldprt format through the interface between ANSYS workbench and Solidworks. Set the material for the gray cast iron. The introduction of 3D model is shown in figure 6:

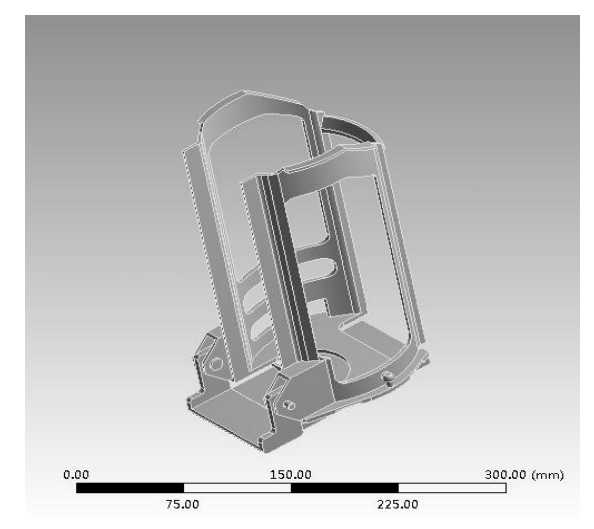

Figure 6. Model Introduction.

(2)In the process of mesh generation, considering the shell cup is irregular, and the structure is more complex, in order to avoid the calculation is too complicated, this method is used to automatically divide the grid, the results are shown in figure 7 :

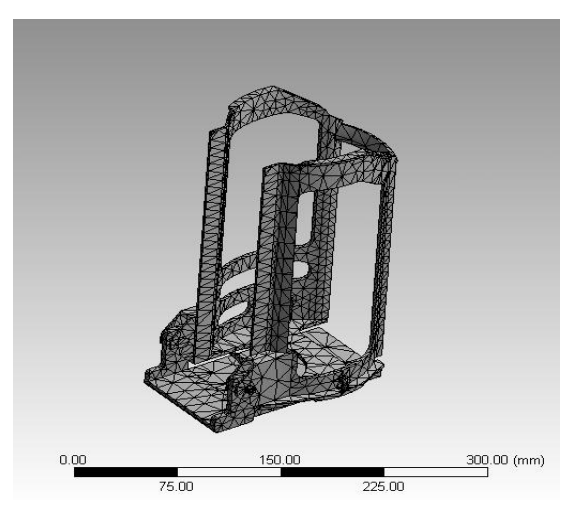

Figure 7. Mesh Graph.

\section{Loading}

The shell cup is applied whole torque $M$ shells on the bottom of the gravity pressure G projectile, and column cylinder spring on the downward spring spring force $\mathrm{F}$, simulated in motion, shell cup and the bottom of the cylinder by two different sizes but the direction perpendicular to the bottom of the upward force of F1 and F2. As shown in figure 8:

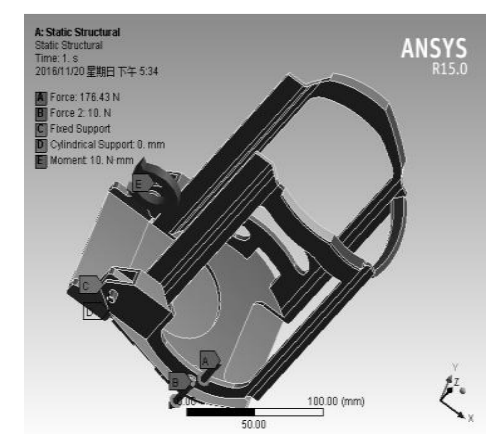

Figure 8. Load Force. 


\section{After-Treatment}

The model is solved and the equivalent stress diagram is used to represent the vibration state of the vehicle, and the results are shown in Figure 9:
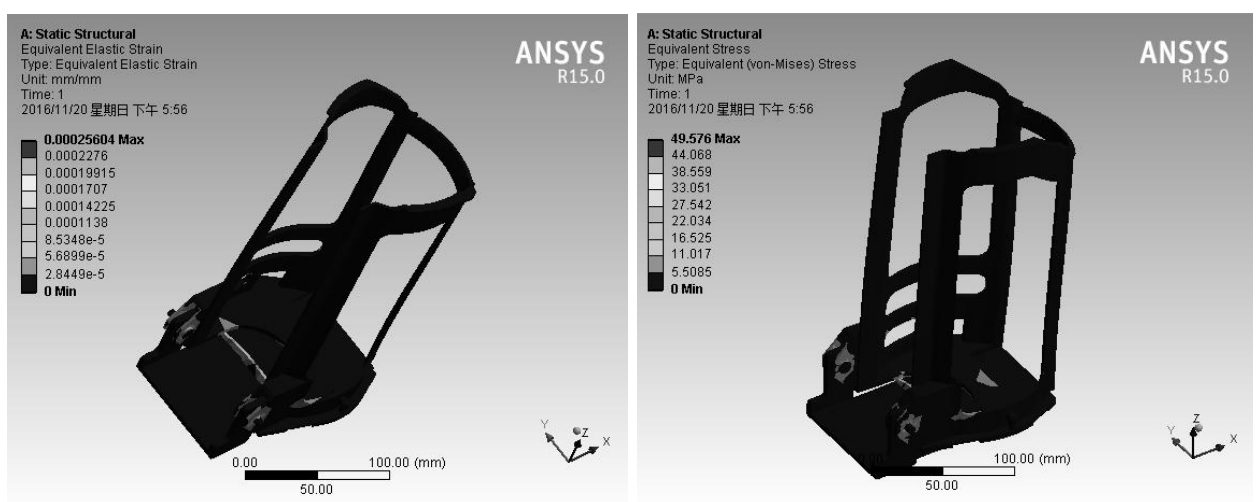

Figure 9. Equivalent Stress and Strain Diagram.

\section{Modal Results}

Modal analysis is an analytic technique to determine the natural frequencies and modal shapes of the components. It is a basic content of other dynamic analysis[4]. A simplified model is established in Solidworks in order to import the Parasolid format into ANSYS, add the relevant constraints, and then carry out modal analysis, and finally get the final results as shown in table 1 and figure 10.

Table 1. Modal Analyze Results of Shell Cup.

\begin{tabular}{|c|c|c|}
\hline Order Number & Natural Frequency & Vibration Characteristic \\
\hline 1 & 133.34 & tube wall inward bending \\
\hline 2 & 152.74 & tube wall to both sides of the varicose \\
\hline 3 & 249.92 & left tilt \\
\hline 4 & 261.04 & expansion deformation in the middle \\
\hline 5 & 335.35 & rear cross beam bending \\
\hline 6 & 488.67 & left rear support rod torsion deformation \\
\hline
\end{tabular}

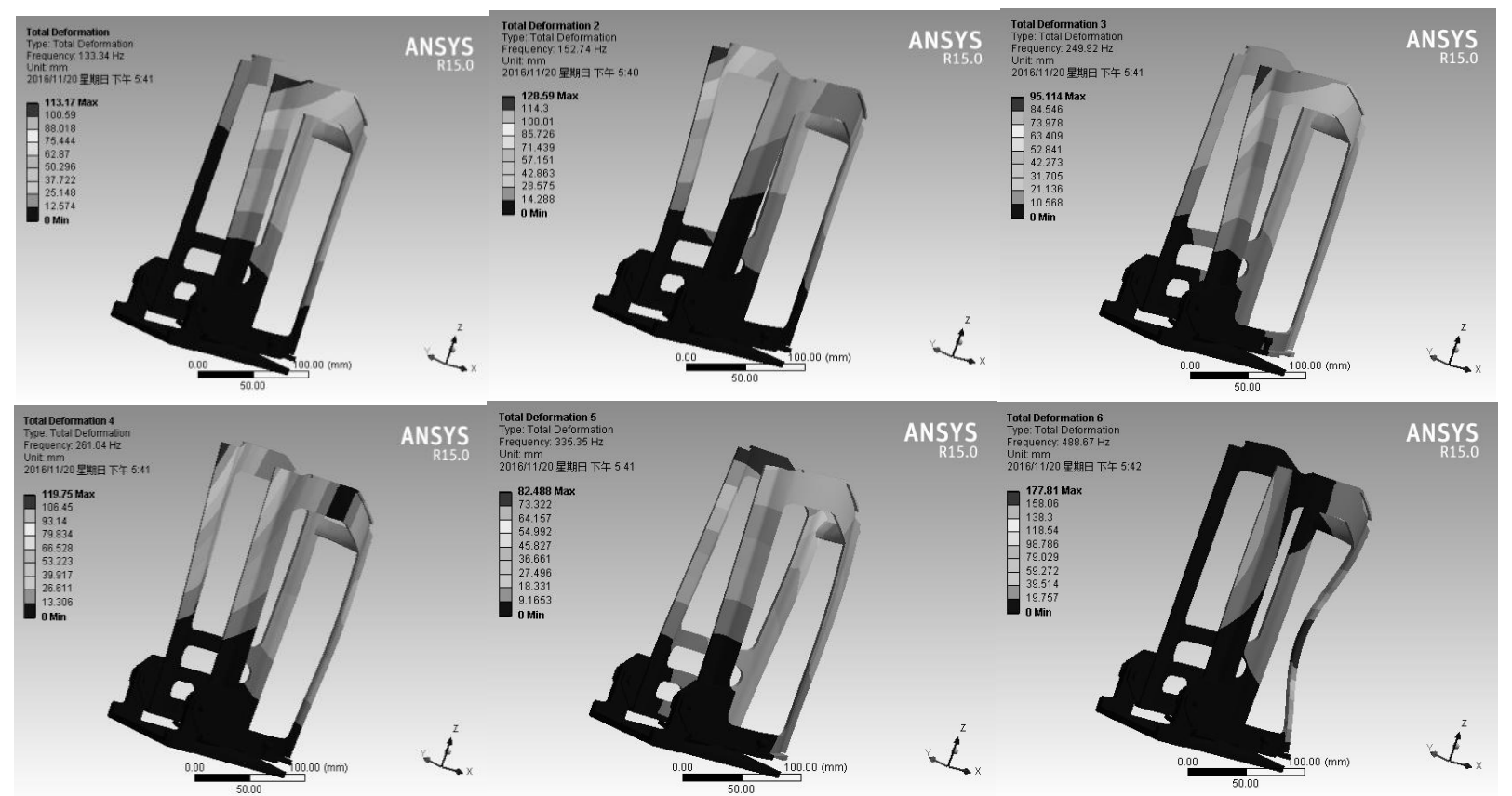

Figure 10. Schematic Diagram. 


\section{Results Analysis and Recommendations for Improvement}

Through the experimental data of the above simulation results, we can draw some conclusions: the shell cup cylinder rear bar is quite easy to break, this point using the actual equipment and problems in the process is the same, should be appropriate to prevent the widening of thickened or similar damage; the cup and the bottom of the cylinder shell under gravity therefore, the cup and the lateral wall of the cylinder welding part is not fast enough, should adopt the integral casting method considering.

\section{Summary}

Through a detailed analysis of a tracked infantry fighting vehicle auto-loader ramming process principle, according to the work state of the shell cup, model was simplified and model in vehicle vibration and steady operation. According to the experimental data analysis of the whole stressstrain conditions and failure mechanism analysis of shell cup, it puts forward some suggestions to improve the optimization, which is for the military sector to a certain extent design and provides reference. The deficiency is not considering the characteristics of rigid flexible coupling bomb device is flexible body and a rigid, rigid flexible coupling simulation realization should be realized in the next step of experiment, in order to get more data and provide more effective and reasonable suggestions.

\section{Reference}

1. Shi-xin Zhang, Yao-hui Zhang, Hui-qiang Lv. Study on the failure regularity and maintenance support scheme of a certain type of infantry combat vehicle [R]. Beijing: The Academy of Armored Forces Engineering, 2011

2. Bao-cheng Ding. A type of infantry fighting vehicle fault diagnosis system of autoloader [J]. Journal of system simulation.2011.23 (9).

3. A certain type of tracked infantry fighting vehicle maintenance guide $[R]$. Beijing: The Academy of Armored Forces Engineering, 2011

4. Wei Li, Ji-sheng Ma, Chang-chun Di. Hydraulic ramming of fault simulation system based on [J]. simulation of 2007, 19 (10).

5. Bao-quan Mao, Guo-hui Wang et al. Modeling and Simulation of vehicle mounted weapons [M]. Beijing: National Defence Industry Press, 2011

6. Bao-quan Mao. Introduction of vehicle weapon technology [M]. Beijing: National Defence Industry Press, 2009

7. Ren-xi Hu, Shi-ting Kang. ANSYS15.0 mechanical and structural finite element analysis from entry to the master [M]. Beijing: Mechanical Industry Press, 2013

8. Jiang Liu. ANSYS mechanical simulation examples [M]. Beijing: Mechanical Industry Press, 2015

9. Jian-hua Luo, Da Xu. Dynamic analysis and Simulation of ammunition loading manipulator based on virtual prototype [J]. Journal of The Academy of Armored Forces Engineering, 2015, (3).

10. Zhong-ting Su, $\mathrm{Da} \mathrm{Xu}, \mathrm{Xiao}-w e i \mathrm{Li}$. A double barrel type infantry fighting vehicle modified [J]. Journal of ballistics, finite element model $(2013,3)$. 